Revue des patrimoines

29 | 2016

Ensembles mobiliers, industriels, techniques.

Connaissance, protection, conservation, présentation au public

\title{
La villa Cavrois : le défi du remeublement
}

The Villa Cavrois: the challenge of refurnishing

\section{Élisabeth Portet}

\section{(2) OpenEdition}

\section{Journals}

Édition électronique

URL : http://journals.openedition.org/insitu/13339

DOI : 10.4000/insitu. 13339

ISSN : 1630-7305

\section{Éditeur}

Ministère de la culture

\section{Référence électronique}

Élisabeth Portet, «La villa Cavrois : le défi du remeublement », In Situ [En ligne], 29 | 2016, mis en ligne le 13 juillet 2016, consulté le 01 mai 2019. URL : http://journals.openedition.org/insitu/13339 ; DOI : 10.4000/insitu.13339

Ce document a été généré automatiquement le 1 mai 2019.

\section{c) (i) $९$}

In Situ Revues des patrimoines est mis à disposition selon les termes de la licence Creative Commons Attribution - Pas d'Utilisation Commerciale - Pas de Modification 4.0 International. 


\title{
La villa Cavrois : le défi du remeublement
}

\author{
The Villa Cavrois: the challenge of refurnishing
}

\section{Élisabeth Portet}

« Demeure pour une famille nombreuse. Demeure pour une famille vivant en 1934 :

air, lumière, travail, sports, hygiène, confort, économie ${ }^{1}$.

Par ces mots, Robert Mallet-Stevens (1886-1945) résume les grands principes du programme architectural de la villa Cavrois qu'il réalise entre 1929 et 1932 pour Paul Cavrois, un industriel du textile, sur les terrains du quartier Beaumont situés à Croix, à la périphérie de Roubaix ${ }^{2}$ (Nord). La demeure est conçue pour abriter une famille de sept enfants et le personnel de service. Elle comprend un pavillon de gardien avec un garage, une piscine, un miroir d'eau, une roseraie, des serres, un potager et un verger répartis sur quatre hectares de terrain. Bien que classique dans sa disposition, la villa s'affiche comme un manifeste esthétique et technique de l'architecture des années 1930. À la fois château des temps modernes ${ }^{3}$ et imposant paquebot recouvert de briques jaunes (fig. 1), la demeure de Paul Cavrois se distingue par le soin apporté aux éléments constructifs et décoratifs, le goût pour une organisation fonctionnelle de l'espace et le dialogue permanent entre l'extérieur et l'intérieur. Chaque pièce est conçue comme un ensemble homogène où le décor et le mobilier affichent une continuité exemplaire pour répondre aux besoins de ses occupants. Robert Mallet-Stevens montre ainsi sa volonté de concevoir la demeure dans ses moindres détails, allant jusqu'à dessiner lui-même le mobilier. Il s'inscrit dans la filiation des recherches de Joseph Hoffmann, d'Henri Van de Velde ou de Frank Lloyd Wright qui renouent avec l'idée d'une "œuvre d'art totale " ou Gesamtkunstwerk. Cette rigueur de conception passe par les matériaux, choisis avec soin, et leur mise en œuvre. 
Figure 1

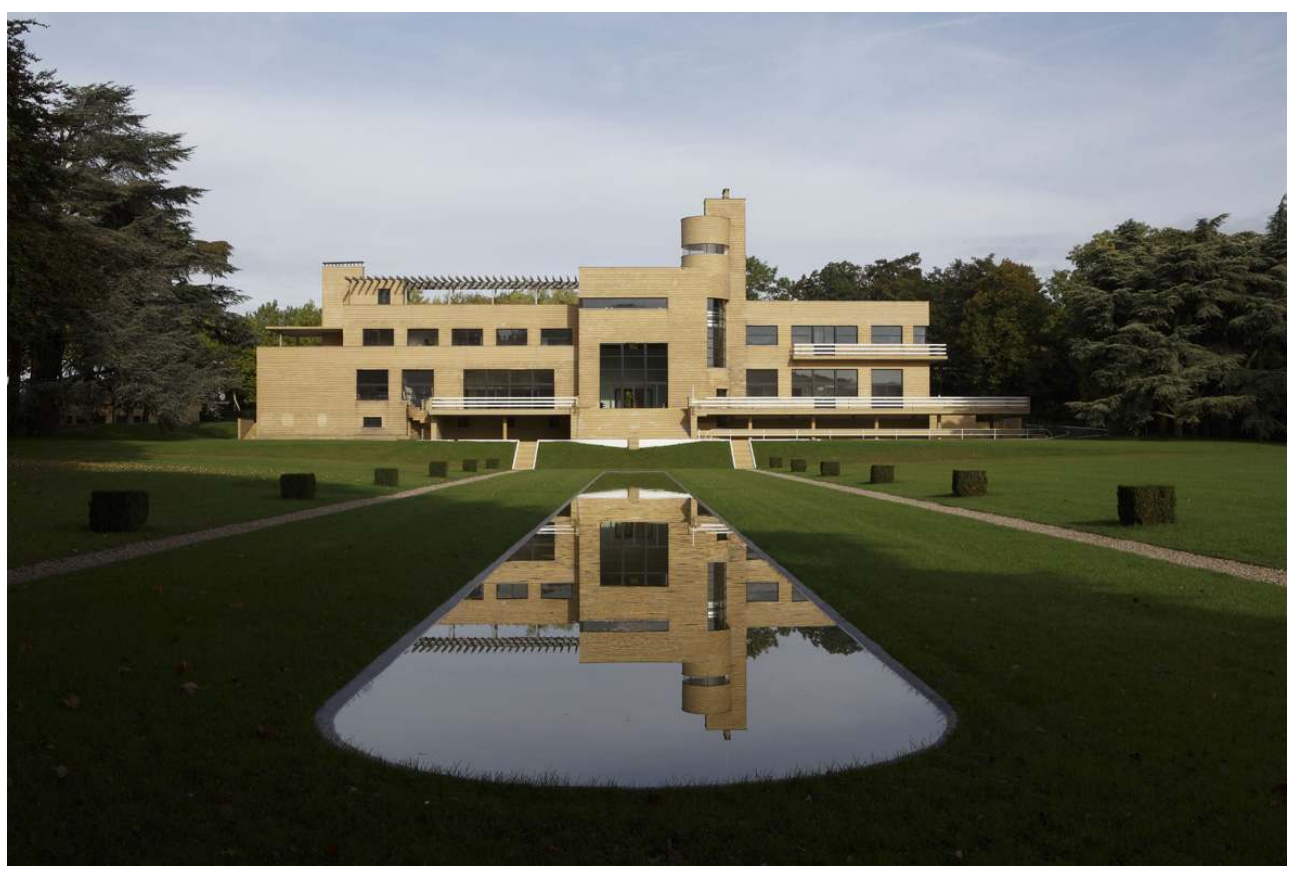

Villa Cavrois, la façade sud dans l'axe du miroir d'eau.

Phot. Paillé, Jean-Luc/Centre des monuments nationaux. ㄷ Jean-Luc Paillé, 2013.

2 Au début des années 2000 , cette réalisation emblématique était devenue une ruine vidée de son mobilier, dépouillée de ses décors et largement amputée de ses jardins dont l'aspect avait perdu toute sa splendeur d'antan. Les campagnes de restauration successives menées depuis 2001 par l'État ont abouti à son ouverture au public le 13 juin 2015. Les visiteurs découvrent aujourd'hui la demeure dans son état historique de 1932, année de sa livraison à la famille Cavrois. Le mobilier d'origine est présenté dans six pièces, disposé d'après des photographies anciennes. Ce résultat est le fruit de longues recherches et l'exploitation d'opportunités uniques; il constitue aussi l'aboutissement d'une réflexion sur le remeublement partiel de la villa afin de retrouver l'esprit qui a guidé sa création dès l'origine.

\section{Histoire d'une renaissance}

\section{L'usage familial de la villa}

L'inauguration de la villa le 5 juillet 1932, à l'occasion du mariage de Geneviève, l'aînée des filles de Paul Cavrois, marque le début d'une histoire mouvementée qui voit se succéder un enchaînement de circonstances malheureuses. Si la famille prend possession des lieux en 1932, elle est contrainte de quitter la demeure, réquisitionnée par l'armée allemande en mai 1940, puis par les Forces françaises de l'Intérieur, elles-mêmes remplacées par une compagnie du train des équipages ${ }^{4}$. Ces événements entraînent quelques dégradations sur les décors et le mobilier mais l'intégrité des aménagements intérieurs est conservée.

Lorsque Paul Cavrois revient dans la villa à la Libération, il souhaite réaliser des transformations pour adapter la demeure à l'évolution familiale. Après le décès de Mallet- 
Stevens en 1945, il demande à l'architecte Pierre Barbe (1900-2004) de remanier la villa en trois appartements indépendants, pour lui-même et son épouse ainsi que ses deux fils aînés, Paul et Francis, avec leur famille. La cohérence des espaces et l'harmonie des décors d'origine sont mises à mal par ces interventions qui s'échelonnent entre 1946 et 1959. Certains meubles sont modifiés pour s'accommoder aux nouveaux usages et aux changements d'affectation des pièces ; toutefois, la plupart sont encore en place lors du décès de Mme Cavrois, en 1985.

\section{La période d'abandon}

En 1985 commence la dégradation de la demeure. Face aux frais d'entretien d'un tel édifice, très importants, et du fait d'une succession complexe, les descendants décident de vendre la propriété puis l'intégralité de son mobilier. La maison est cédée en 1986 à une société immobilière tandis que le mobilier est dispersé aux enchères le 5 avril 1987 à Monaco (Sotheby's). Pour son nouveau propriétaire, la villa et l'ensemble de ses terrains présentent des opportunités de lotissement dans un quartier très convoité. Un projet de construction de plusieurs petits immeubles et d'une résidence de luxe implantée dans la villa est envisagé mais le permis de construire est rejeté par la mairie de Croix en 1988. Entretemps, le 9 décembre 1987, la villa est inscrite à l'inventaire supplémentaire des monuments historiques, pour sauver ce qui peut encore l'être.

Obligé de renoncer à son projet, le propriétaire laisse la villa à l'abandon. Livré au pillage, vandalisé et dégradé, l'édifice est gravement menacé (fig. 2). Pour faire face à cette situation, le ministère de la Culture prend une instance de classement qui aboutit à une décision de classement d'office au titre des monuments historiques le 12 décembre $1990^{5}$. Malgré cette mesure exceptionnelle, la villa continue de se dégrader rapidement du fait de l'absence d'entretien et de l'occupation de squatteurs. Après des tentatives de négociation avec le propriétaire, restées sans succès, les pouvoirs publics exigent en 1997 la réalisation de travaux de sauvegarde. Il s'ensuit une bataille judiciaire qui aboutit en 2001 à la décision d'achat de la villa en péril par l'État, avec une partie du parc. 
Figure 2

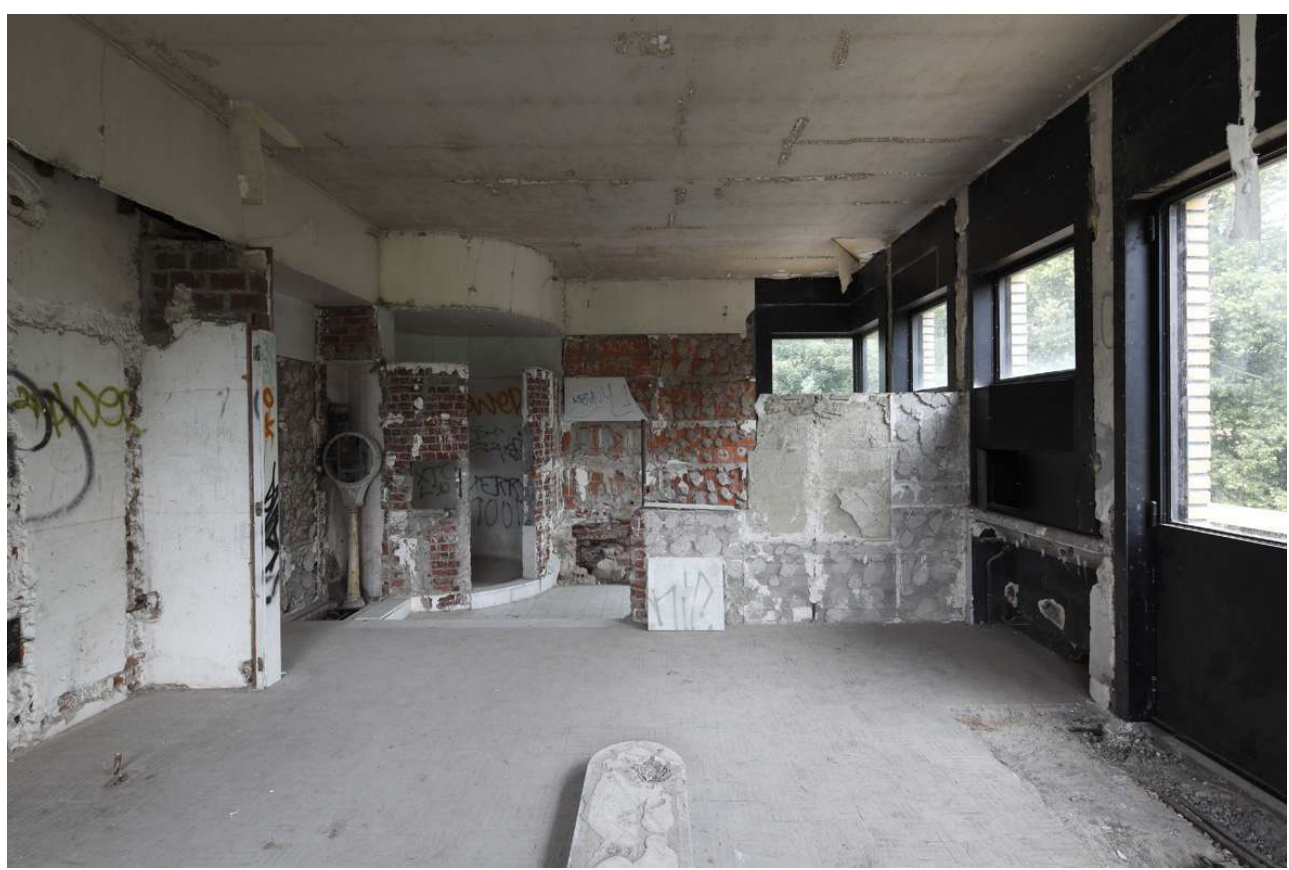

Villa Cavrois, la salle de bain des parents

Phot. Paillé, Jean-Luc/Centre des monuments nationaux. (c) Jean-Luc Paillé, 2012.

\section{La restauration}

7 L'acquisition de la demeure par l'État met fin à une longue période d'incertitude sur le devenir du monument. Elle marque le début de la réhabilitation de l'édifice qui connaît plusieurs phases de travaux sur une durée totale de quinze ans ${ }^{6}$. Une première campagne (2001-2002) réalisée par l'architecte des Bâtiments de France, Pierre Cusenier, sous la maîtrise d'ouvrage de la direction régionale des Affaires culturelles Nord-Pas-de-Calais Picardie (DRAC), a consisté à garantir la sécurité du site et contenir la dégradation de l'édifice. La deuxième campagne (2003-2015), menée par l'architecte en chef des Monuments historiques Michel Goutal, s'est attachée à la restauration du clos et du couvert, du parc puis des intérieurs, dont le principe repose sur la restitution des espaces et des volumes d'origine afin de se rapprocher de l'état historique de $1932^{7}$. D'abord assurée par la DRAC, la maîtrise d'ouvrage a été reprise au début de l'année 2009 par le Centre des monuments nationaux qui a conduit l'achèvement des travaux. Le vaste chantier de restauration du parc et des espaces intérieurs s'est déroulé sur deux tranches concernant le corps central de la villa dans un premier temps, puis les ailes et le pavillon du gardien dans un second temps.

\section{Le projet de remeublement}

\section{L'inventaire des collections}

Alors que le démarrage des travaux de restauration du bâtiment permet d'envisager un nouvel avenir pour la villa, différentes hypothèses de réutilisation sont évoquées dès 
1989. Projet de centre d'art, dépôt du FRAC, conservatoire de l'architecture contemporaine ou centre d'archives régionales de l'architecture moderne, les premières pistes de réflexion privilégient les partenariats avec les acteurs locaux comme l'école d'architecture ou le Centre des archives du monde du travail à Roubaix et les acteurs nationaux comme l'Institut français d'architecture ${ }^{8}$. La remise en dotation de la villa Cavrois au Centre des monuments nationaux le 18 décembre 2008 relance la question du projet culturel et scientifique du monument en vue de son ouverture au public.

Le travail d'inventaire et de recensement du mobilier a débuté dans ce contexte. En l'absence de fonds d'archives concernant Mallet-Stevens', les sources imprimées et les campagnes photographiques de l'époque ont constitué les seules ressources documentaires disponibles. L'ouvrage Une Demeure $1934^{10}$ (fig. 3), le catalogue de l'exposition « Rob Mallet-Stevens. Architecture, mobilier, décoration ${ }^{11}$, qui s'est tenue à Paris, Villeneuve-d'Ascq, Bruxelles et Tours en 1986, la monographie de Richard Klein ${ }^{12}$ et le catalogue de l'exposition "Robert Mallet-Stevens, l'œuvre complète " ${ }^{13}$ organisée au Centre Pompidou en 2005 ont permis de mieux connaître le contexte de réalisation du mobilier de la villa. Les fonds photographiques conservés au musée des Arts décoratifs et au Centre canadien d'architecture de Montréal ${ }^{14}$ offrent une vision des intérieurs meublés de la villa en 1932. Ces vues d'époque ont été confrontées aux clichés en couleur pris au moment de la vente de la maison, en 1986, par Véra Cardot et Pierre Joly, dont le fonds est conservé au Centre Pompidou. Les photographies de l'intérieur de la maison lors de sa livraison à la famille Cavrois ont servi de point de départ à l'élaboration de l'inventaire des collections. Si l'angle de vue adopté par les photographes limite l'étude aux objets visibles sur les clichés, l'ample couverture photographique de la villa constitue un outil précieux pour dresser une première liste du mobilier dans chaque pièce. En revanche, l'absence d'images concernant les zones techniques laisse de nombreuses incertitudes, parfois levées par l'observation des plans qui indiquent l'implantation au sol du gros mobilier. 


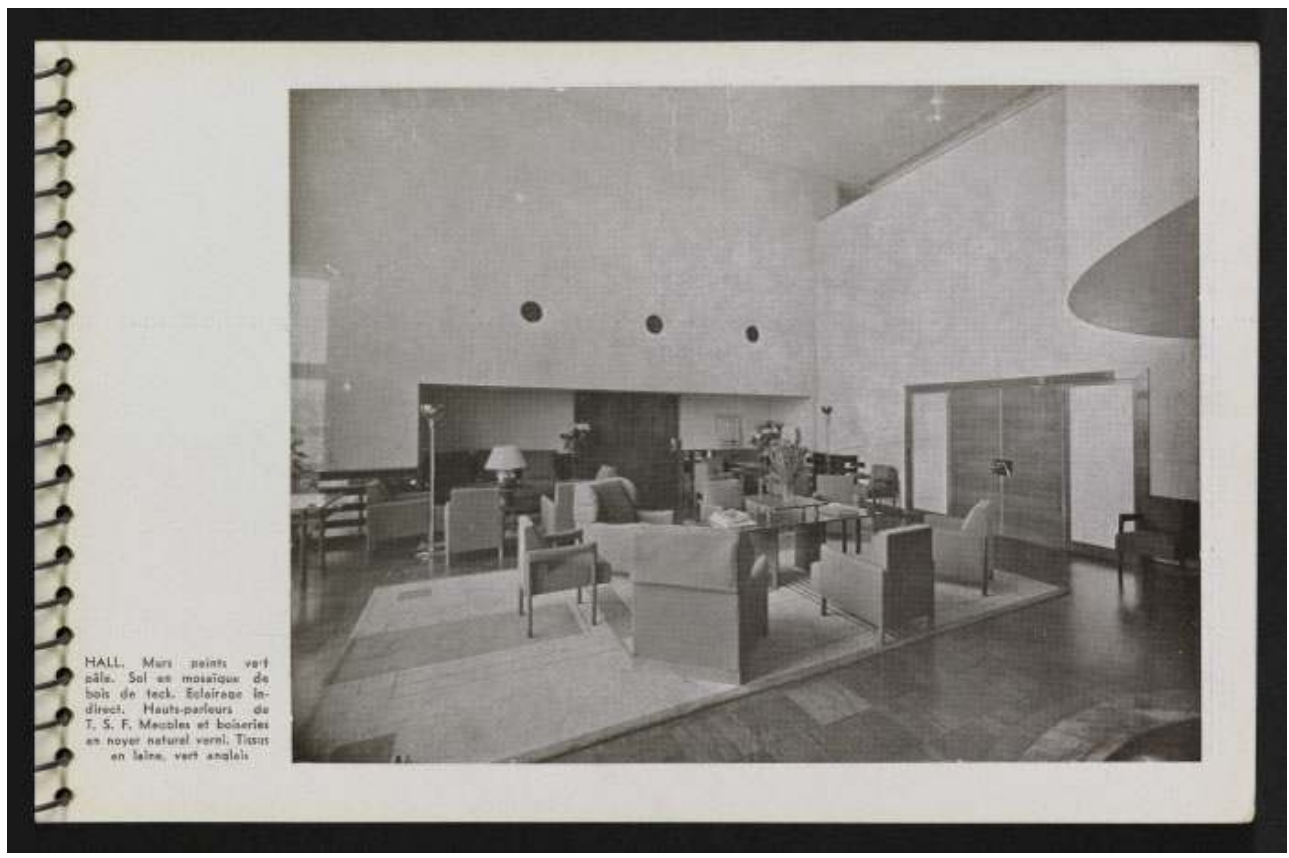

Villa Cavrois, vue intérieure du hall publiée dans Une Demeure 1934.

Phot. Berthé, Philippe/Centre des monuments nationaux. (c) Philippe Berthé, 2011.

Les archives de Pierre Barbe, versées au Centre d'archives d'architecture du xxe siècle, constituées de plans, coupes et relevés du mobilier de la villa, ont été consultées pour étudier l'impact des interventions de l'architecte sur les meubles dessinés par MalletStevens. Le premier résultat de ce travail d'investigation a été la confirmation de la part importante de l'action de Pierre Barbe sur les modifications du mobilier dans les années 1950. Les meubles s'adaptent à l'évolution familiale et au nouveau cloisonnement des espaces, qui nécessite une réduction de leur taille. C'est ainsi que Barbe conserve les portes des buffets ou des bahuts mais en modifie le bâti pour mieux les insérer dans les pièces transformées. Par exemple, le mur formant bibliothèque du bureau de Paul Cavrois, constitué d'une rangée de placards bas à six portes et de deux étagères parfaitement intégrées dans le décor des lambris, visible sur les photographies de 1932, est devenu un meuble-bibliothèque indépendant. Le champ d'action de Barbe couvre la totalité des pièces et des aménagements intérieurs, du décor aux meubles meublants, à l'image de la coiffeuse du boudoir pour laquelle il rajoute un tiroir central sous le plateau (fig. 4). Si certains meubles ont été légèrement modifiés, d'autres font l'objet de transformations radicales, comme la grande table de la salle à manger des parents, initialement de forme rectangulaire en poirier noirci et laqué, devenue une table ronde pouvant accueillir quatre rallonges avec plateau en noyer blanc laqué ${ }^{15}$. Dans d'autres cas, le mobilier d'origine disparaît intégralement au profit d'une réalisation nouvelle qui en conserve toutefois l'usage initial. Ainsi, les banquettes en maroquin du coin du feu dans le hall sont remplacées par deux canapés recouverts de velours. 


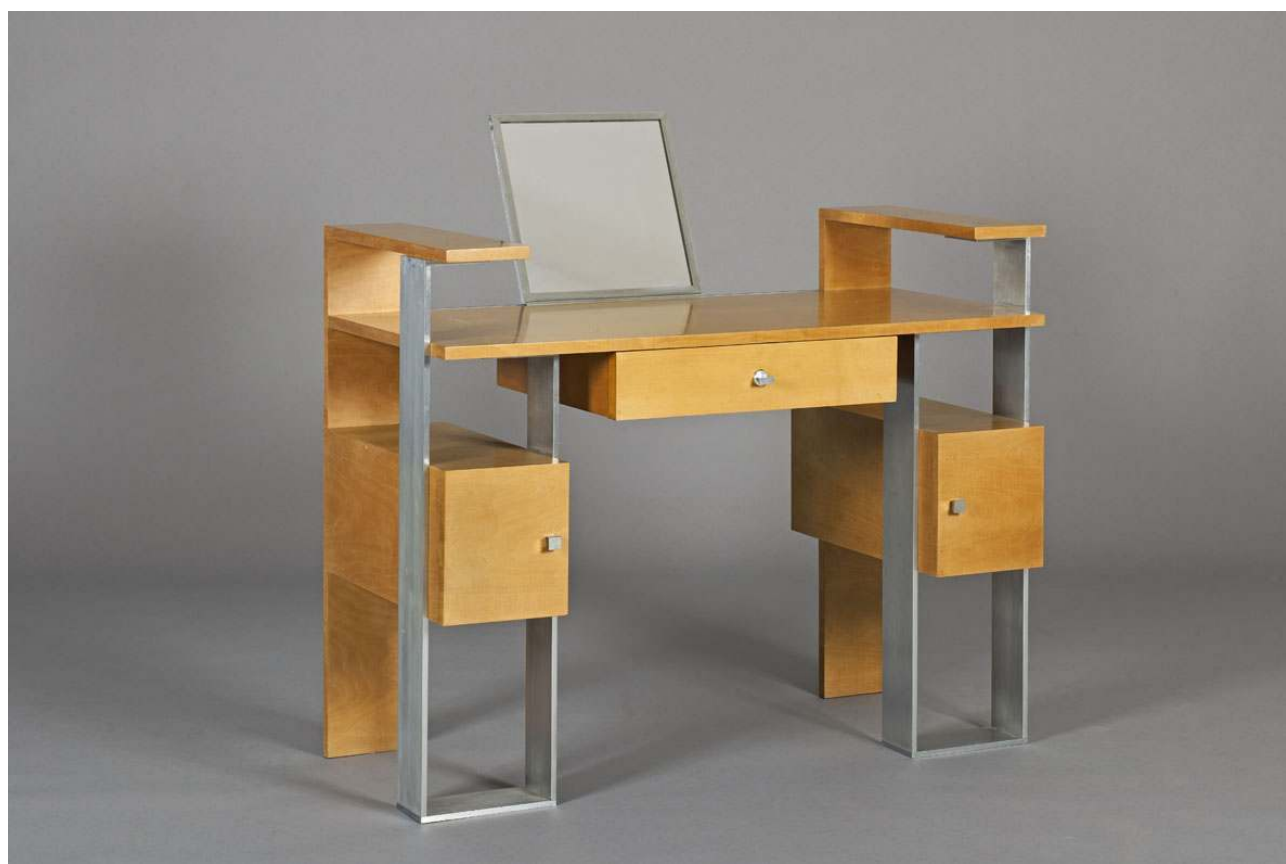

Villa Cavrois, coiffeuse du boudoir.

Phot. Cadet, Patrick/Centre des monuments nationaux. ( Patrick Cadet, 2012.

\section{Repérage et localisation du mobilier}

11 Une fois ce panorama esquissé, l'identification et la localisation des biens ont été menées à partir des ventes successives du mobilier de la maison depuis leur dispersion, à l'occasion de la première cession du 5 avril 1987. Le catalogue ${ }^{16}$ répertorie 42 lots provenant de différentes pièces de la villa, dont le mobilier de jardin. Malgré la provenance des objets, cette vente est loin d'être un succès. Deux explications à ce constat: d'abord, la reconnaissance de ce type de meubles, alors considérés comme " d'avant-garde », tarde à venir, ensuite, la transformation de certains meubles par Barbe a compliqué leur attribution, semant le trouble chez les acquéreurs potentiels. Il faut aussi noter l'aspect très incomplet de la vente par rapport au nombre d'objets présents dans la villa car une partie du mobilier a été vendue de gré à gré, l'année précédente, par les descendants Cavrois.

Désormais dispersés, les meubles attirent l'attention d'un public d'experts. Acquis par des galeristes reconnus et revendus successivement à des collectionneurs avertis, ils réapparaissent parfois dans les ventes publiques ; c'est le cas du meuble d'appui du hall qui passe en vente à New York en juin 1996 puis de nouveau en décembre $2000^{17}$. Les ensembles mobiliers de la salle à manger des enfants, du boudoir, les sièges du hall et les tabourets des salles de bain errent longtemps sans trouver amateur avant d'entrer dans la collection de Karsten Greve ${ }^{18}$ qui les remet en vente aux enchères à Paris en 2003 en deux vacations $^{19}$ dont les résultats d'adjudication atteignent des records. Dans ces années 2002-2003, l'incertitude sur le projet de réutilisation de la villa ne permet pas de concrétiser les acquisitions de ces ensembles dont certains font l'objet, auprès du 
ministère de la Culture et de la Communication, de demandes de certificats de libre circulation en vue de leur exportation ${ }^{20}$.

Les recherches se sont poursuivies auprès des maisons de vente et des galeristes pour retrouver la trace des autres meubles. L'aide et le soutien apportés par les descendants de Mallet-Stevens puis par la famille Cavrois ont permis de lever de nombreuses incertitudes. À l'issue des investigations, à la fin de l'année 2009, il se confirme que la majeure partie du mobilier se trouve en mains privées. Seuls deux meubles sont conservés dans des musées: le chevet d'une des chambres de jeune homme du rez-dechaussée, au Vitra Design Museum à Weil-am-Rhein (Allemagne) et une table de cuisine, au musée des Années 30 de Boulogne-Billancourt. Pour achever ce panorama, une dizaine de biens sont alors en vente sur le marché de l'art.

\section{Le parti pris de restauration et les choix de restitution}

L'état des lieux dressé souligne l'ampleur de la tâche et la difficulté de réunir un ensemble mobilier dispersé. Alors que les collections sont disséminées chez les particuliers et qu'une partie n'a toujours pas été localisée, comment envisager le remeublement de la villa Cavrois? Cette question surgit au moment où la commission nationale des Monuments historiques prend le parti, le 6 décembre 2010, de restituer la villa dans son état d'origine de 1932 avec l'ensemble de ses décors. Au regard de la conception homogène de la demeure, ce choix de restauration signifie la réalisation à l'identique, dans le respect des matériaux d'origine, d'un grand nombre d'éléments liés à l'immeuble comme les luminaires d'applique, les meubles étagères, les banquettes et les meubles de rangement intégrés aux lambris ainsi que les équipements de plomberie et de chauffage. Dans le même temps, l'acquisition du mobilier d'origine devient un axe prioritaire afin de compléter les aménagements intérieurs ${ }^{21}$.

\section{La constitution des collections}

\section{L'acquisition des ensembles mobiliers d'origine}

Une fois définie, la mise en œuvre du projet se heurte à une double difficulté, celle de la disponibilité d'un mobilier qui a une cote désormais élevée sur le marché de l'art mais dont l'acquisition doit tenir dans une enveloppe budgétaire raisonnable, et celle du maintien de l'unité des ensembles.

La vente des collections du château de Gourdon (Alpes-Maritimes) en $2011^{22}$ marque le début d'une politique d'acquisition importante. Les trois vacations des 29 mars, 29 et 30 juin ont permis au Centre des monuments nationaux d'acquérir pour le compte de l'État, avec l'aide du fonds du Patrimoine, les meubles du boudoir de Mme Cavrois comprenant la coiffeuse, la travailleuse, la pendule, le fauteuil et la paire de chauffeuses ainsi que trois appliques du vestibule et un meuble de rangement.

Réalisé en placage de sycomore et aluminium poli, le mobilier du boudoir est conçu pour une pièce intimiste située au premier étage de la villa et attenante à la chambre parentale. Il se caractérise par l'utilisation de formes géométriques simples qui mêlent l'aspect traditionnel $\mathrm{du}$ bois à l'aluminium poli, matériau résolument moderne. L'esthétique pure de ce mobilier n'en exclut pas pour autant une approche fonctionnelle dans la justesse des proportions et des accessoires. Le miroir de la coiffeuse est ajustable, 
les casiers de la travailleuse sont pourvus de boîtes de rangement de différents formats. Les sièges affichent des volumes simples où la précision du tracé s'adapte à la configuration de la pièce. La pendule, avec son cadran rectangulaire en miroir posé sur un socle demi-circulaire en laiton, affiche une grande simplicité. Cette rigueur se retrouve dans le traitement des chiffres du cadran au graphisme épuré qui reprend celui des horloges encastrées dans les murs de chaque pièce. Il s'agit d'un mobilier d'architecte qui privilégie les volumes à l'ornement. À l'image des autres meubles de la maison, les créations du boudoir témoignent de la modernité de l'approche de Mallet-Stevens, alors président de l'Union des artistes modernes, et rappellent son admiration pour les recherches du mouvement hollandais de Stij ${ }^{23}$.

Lors des journées européennes du patrimoine de 2013, l'exposition de la travailleuse (fig. 5) dans les salons du ministère de la Culture à l'occasion du centenaire de la loi de 1913 relative aux monuments historiques ${ }^{24}$ a permis de montrer en avant-première aux visiteurs cette recherche constructive et fonctionnelle.

Figure 5

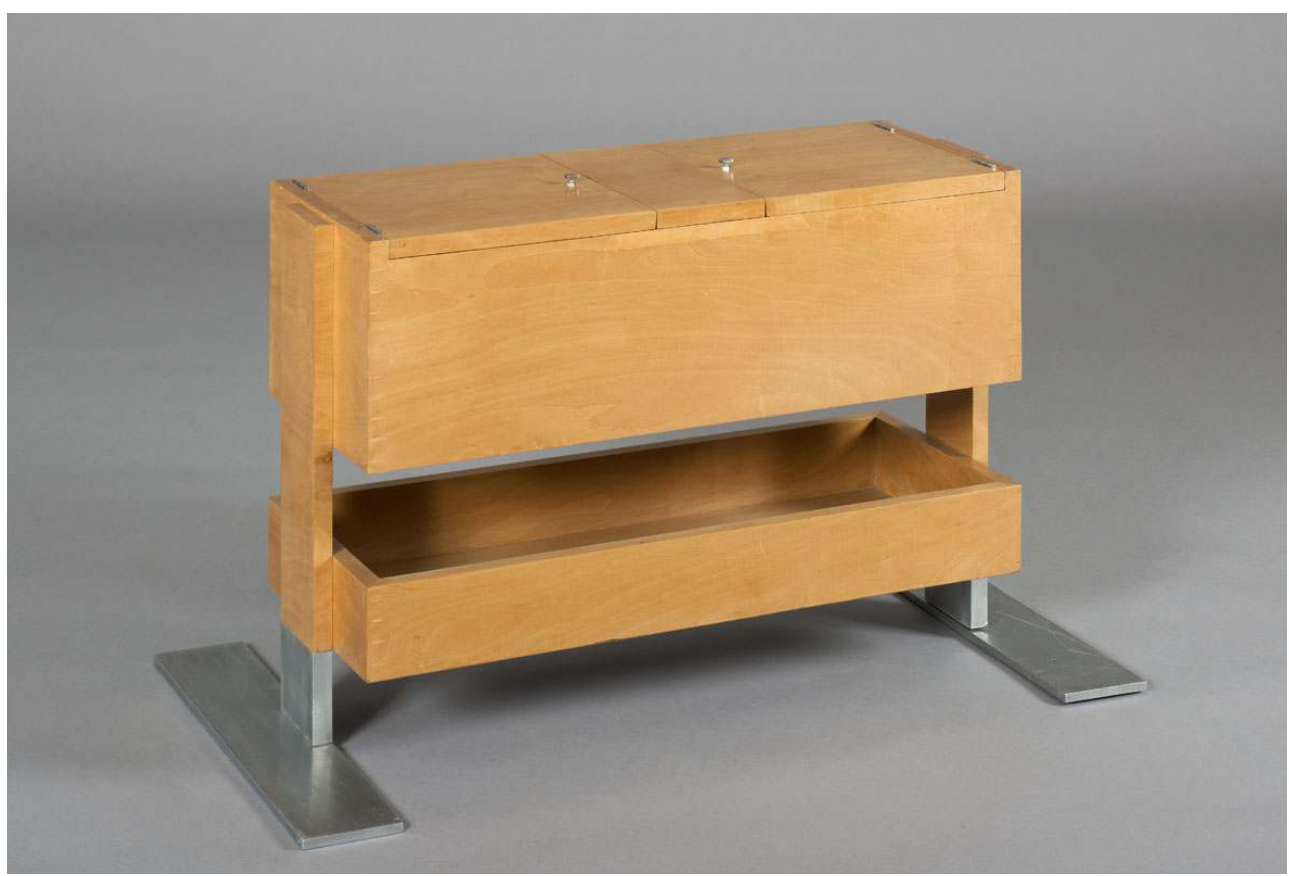

Villa Cavrois, travailleuse du boudoir

Phot. Berthé, Philippe/Centre des monuments nationaux. @ Philippe Berthé, 2012.

Les appliques du vestibule (fig. 6) reflètent un autre aspect du travail de Mallet-Stevens, celui sur la lumière, indissociable de son activité de décorateur de cinéma qui l'occupe entre 1920 et 1928. L'éclairage de la demeure est une préoccupation centrale de l'architecte qui exploite toutes les possibilités de la lumière électrique. Placées de part et d'autre de l'entrée, près d'un miroir d'angle, les appliques s'apparentent à de véritables sculptures. Si Mallet-Stevens conçoit l'ensemble des accessoires et du mobilier de la villa, il fait aussi appel à des collaborateurs qu'il considère comme des associés. Jacques Le Chevallier, décorateur verrier et René Koechlin ${ }^{25}$, ingénieur passionné de mécanique automobile, sont les auteurs des appliques aux cerceaux en aluminium poli formant des bandeaux superposés en décroché et fixés sur une plaque d'ébonite. Les matériaux issus de l'industrie, aux visseries visibles, sont soumis aux lignes géométriques épurées 
interdisant toute ornementation pour répondre à une logique constructive. Participant à l'esthétique moderne de la demeure, ces appliques diffusent une lumière multidirectionnelle qui contribue à la mise en scène de l'architecture dès l'entrée.

Acquis dans la même vente, un meuble de rangement à trois portes ouvrant sur des tiroirs à chemises révèle l'approche rationnelle de l'architecte dans la simplicité des formes géométriques. Chaque tiroir coulisse aisément et rappelle l'importance accordée à l'aspect pratique du meuble, destiné à l'entretien du linge et à son rangement. Il vient ainsi compléter les nombreux placards intégrés sur toute la hauteur dans les chambres et les pièces de service.

Figure 6

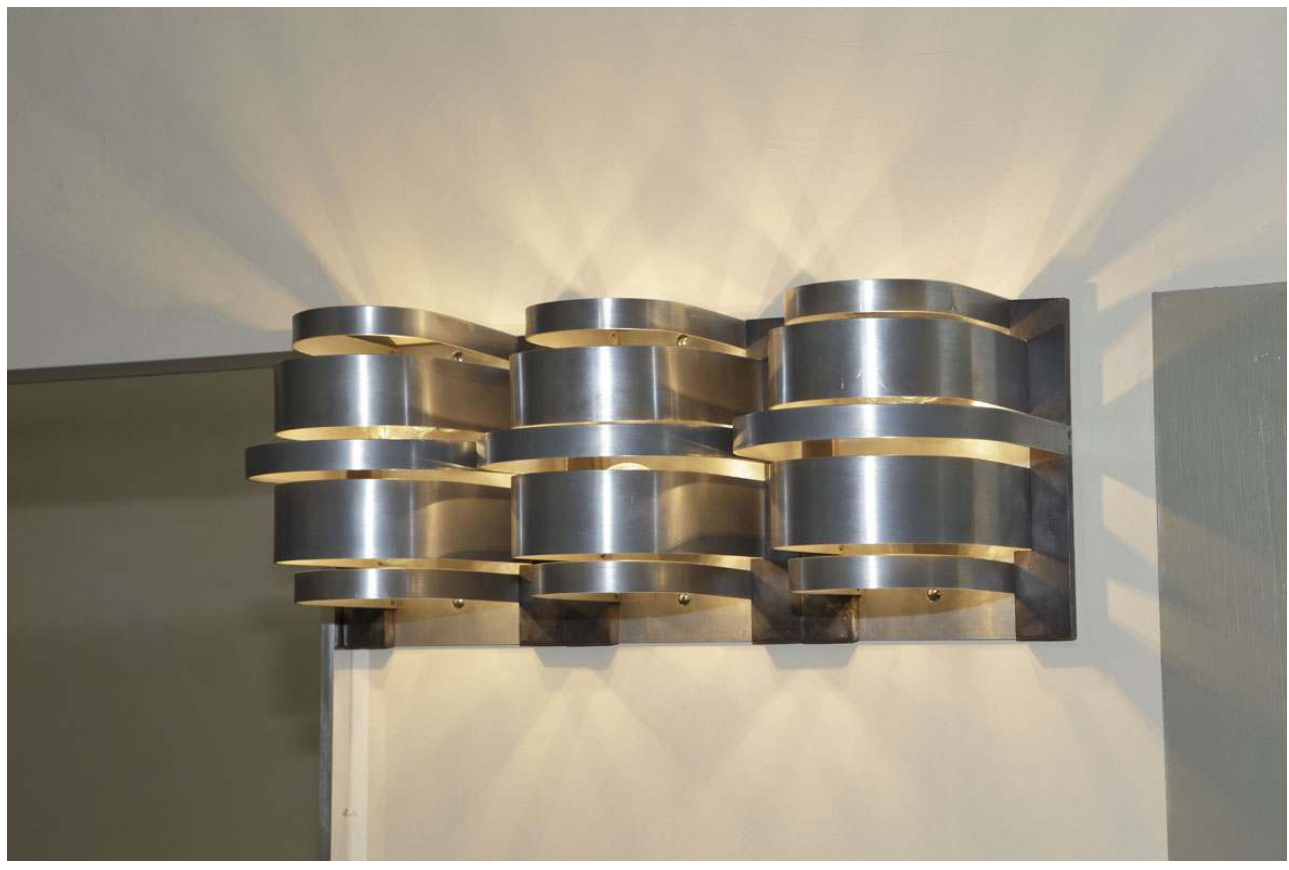

Villa Cavrois, rez-de-chaussée, applique du vestibule.

Phot. Paillé, Jean-Luc/Centre des monuments nationaux. ㄷ Jean-Luc Paillé, 2013.

21 Par chance, une autre vente aux enchères, le 6 juin $2012^{26}$, à Paris, permet de poursuivre l'enrichissement des collections avec l'acquisition de la table et des six chaises de la salle à manger des enfants puis de la paire de fauteuils de la chambre des parents. Le mobilier de la salle à manger en placage de zingana vient compléter la décoration soignée de la pièce aux lambris du même bois exotique, dans une volonté d'unité. La table se compose d'un épais plateau rectangulaire et d'un piétement formé de six panneaux ajourés reposant sur une base décorative débordante plaquée de feuilles d'aluminium. Les chaises (fig. 7) présentent des pieds de section carrée en sycomore alors que le dossier bandeau et l'assise sont plaqués de zingana. Encore une fois, les formes géométriques sont affirmées et traduites par l'utilisation d'essences raffinées avec le zingana, associée à des matériaux industriels comme les feuilles d'aluminium aux visseries apparentes. La paire de fauteuils de la chambre parentale témoigne d'une double volonté de raffinement et de confort. Le placage de bois de palmier contribue à l'élégance du siège tandis que la forme révèle un dossier large, une assise profonde et des accoudoirs à manchette plate. Les pieds avant sont chaussés de manchons en laiton patiné, nouvelle association du bois avec le métal, omniprésente. Le choix du placage en palmier, plutôt foncé, permet à 
l'architecte de jouer sur les contrastes de tons avec les couleurs claires des murs, peints en beige.

Figure 7

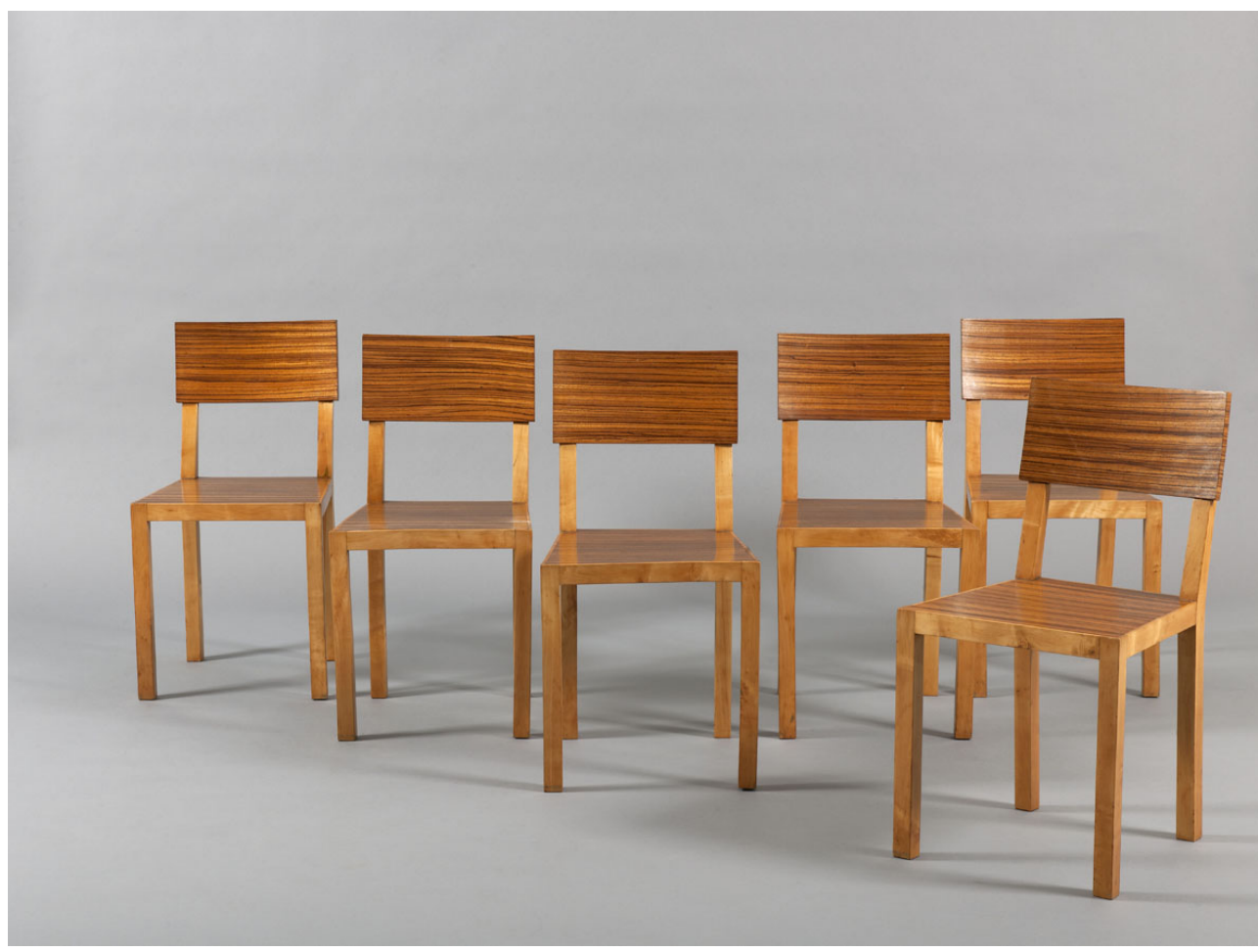

Villa Cavrois, chaises de la salle à manger des enfants.

Phot. Cadet, Patrick/Centre des monuments nationaux. (c) Patrick Cadet, 2012

La vente de la collection d'Alain Braunstein, le 23 mai $2013^{27}$ à Paris, fournit l'occasion d'acquérir L'Hermine, sculpture des frères Jan et Joël Martel en pierre reconstituée. L'œuvre, au dessin épuré et aux formes stylisées, évoque l'importance de la collaboration artistique entre les sculpteurs et l'architecte. Les Martel contribuent activement à la décoration de la villa en intégrant plusieurs sculptures dont un bas-relief polychrome sur le thème du jeu dans la salle à manger des enfants, un Chat assis dans le fumoir de Paul Cavrois et un Pigeon dans le hall.

Ces acquisitions sont complétées en 2014 par le don de deux tables de cuisine en acier tubulaire et d'un ensemble de placards courbes provenant de l'office. Les deux tables, dotées de tiroirs sur la ceinture, présentent un piétement métallique à entretoise sur lequel repose un plateau rectangulaire en faïence carrelé. Les placards suivent la courbe du mur de l'office, où ils sont disposés sur deux rangées parallèles en partie haute et basse. Leur acquisition a permis leur ré-intégration dans la pièce au lieu de la réfection à l'identique prévue dans le projet initial.

\section{La recherche « d'équivalents »}

En parallèle, une réflexion s'engage pour compléter les espaces par du mobilier équivalent. Très ciblées en raison du caractère spécifique de la villa, les acquisitions portent sur le mobilier de série et les petits objets de décoration. 

salle de jeux des enfants avec les chaises de Marcel Breuer éditées par Thonet, dans le jardin et dans les pièces de service. C'est ainsi que deux suites de trois fauteuils et huit chaises en acier tubulaire, d'éditions postérieures à l'original, ont été acquises à Paris le 15 juin $2009^{28}$. Les fauteuils rappellent le mobilier de jardin. Leur conception relève d'un mobilier empilable, ergonomique et pratique dont la fabrication en série répond aux besoins du plus grand nombre. Les chaises ${ }^{29}$, d'un modèle similaire à celles visibles sur les photographies anciennes, complètent l'aménagement de la cuisine et l'office.

À côté de cette production de série, un petit nombre d'accessoires issus du commerce parachève la décoration. Les pièces d'orfèvrerie, les cendriers et vases présents dans la maison sont fournis par les firmes en vogue dans les années 1930 comme DIM (Décoration Intérieure Moderne), la maison Desny ou la cristallerie Baccarat. Les vues du boudoir de 1932 montrent que deux bougeoirs en cristal étaient placés sur la cheminée du boudoir, de chaque côté de la pendule. Dans la perspective de la garnir à nouveau, quatre bougeoirs (fig. 8) en cristal Baccarat rejoignent les collections de la villa Cavrois en 2012. Composés d'une base rectangulaire et d'un fût carré, ils complètent la présentation des ensembles mobiliers acquis pour le boudoir.

Figure 8

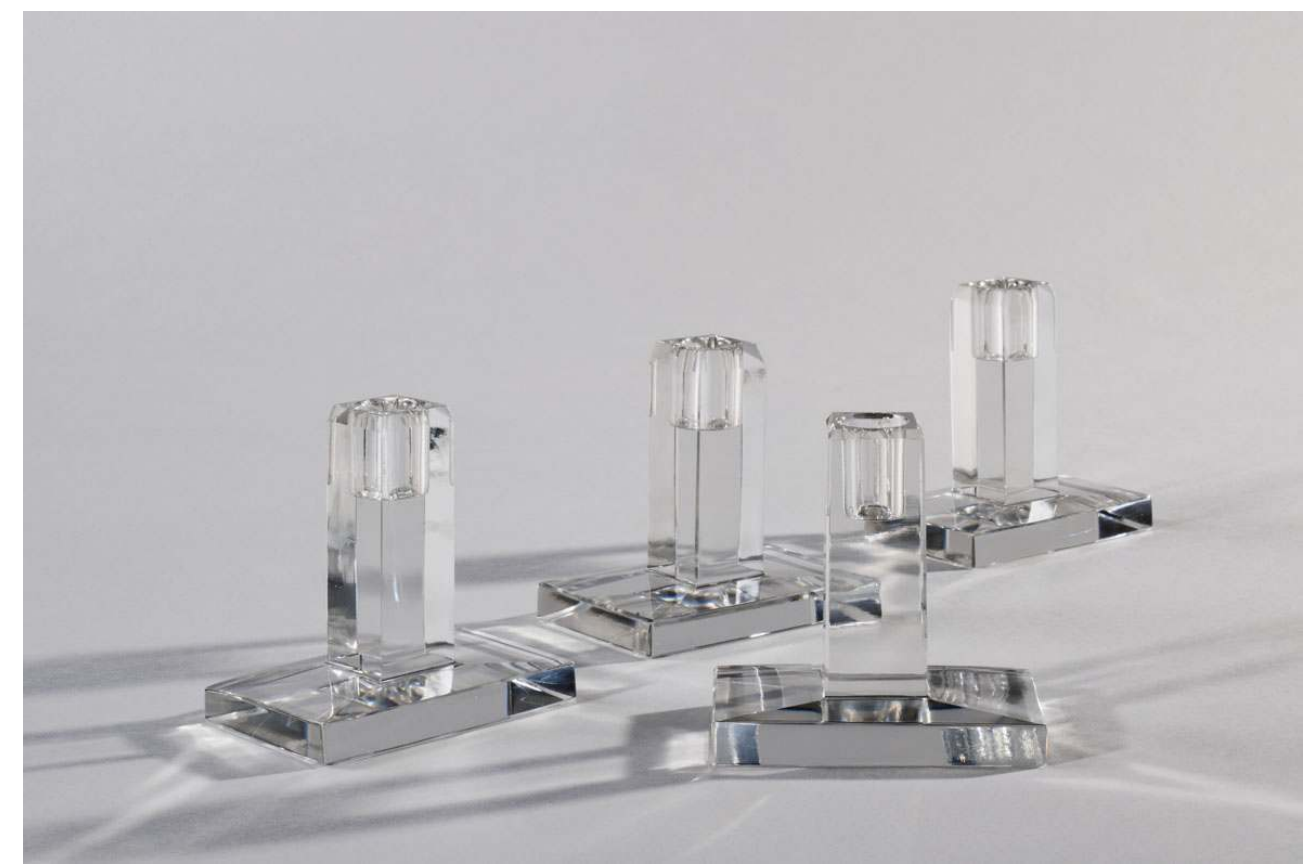

Villa Cavrois, quatre bougeoirs en cristal destinés au boudoir.

Phot. Cadet, Patrick/Centre des monuments nationaux. (c) Patrick Cadet, 2013.

\section{La présentation des collections}

\section{La disposition du mobilier}

Les photographies d'époque donnent une idée assez précise de la façon dont les pièces étaient aménagées en 1932. L'étude de ces documents a naturellement guidé la disposition des meubles dans la maison. Chaque élément mobilier est placé selon la 
configuration d'origine avec des adaptations pour faciliter le passage du public et la sécurisation des collections. C'est la raison pour laquelle les visiteurs ne rentrent pas dans le boudoir. Seules L'Hermine et une table de cuisine ont changé d'emplacement. En effet, la sculpture des frères Martel (fig. 9) est exposée dans la salle à manger des parents, et non au coin du feu du hall où elle figurait à l'origine, sur le rebord de la cheminée.

Figure 9

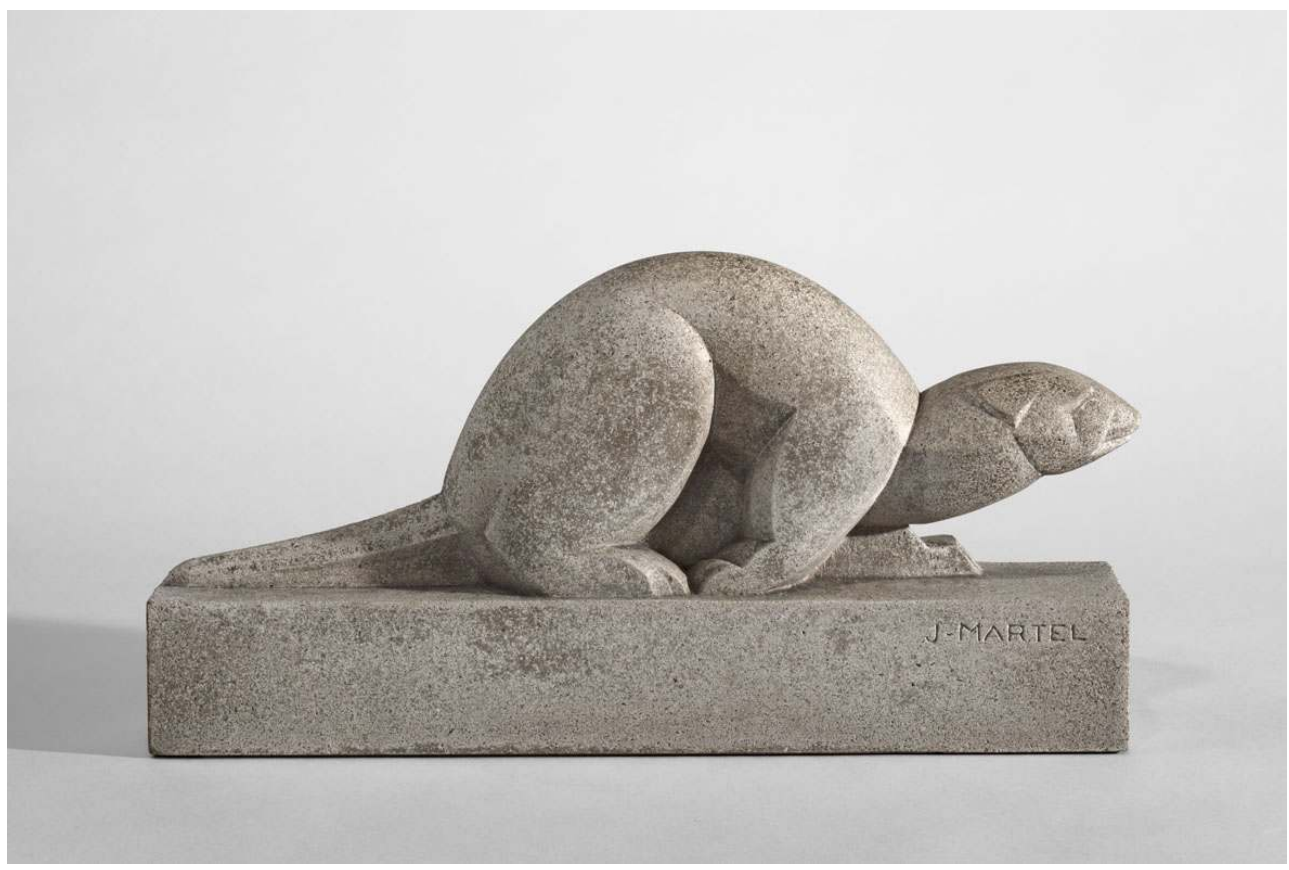

Villa Cavrois, L'Hermine du hall par Jan et Joël Martel.

Phot. Berthé, Philippe/Centre des monuments nationaux. (c) Philippe Berthé, 2013.

Une des trois tables de cuisine est présentée dans la matériauthèque située dans les caves à vin avec l'échantillonnage des vestiges collectés sur le chantier ${ }^{30}$. Ce choix de présentation s'est imposé en raison de son état de conservation. Cette table, très altérée, a été miraculeusement retrouvée dans les caves de la villa, sauvée de la destruction. Hormis les rares équipements techniques encore en place et les fragments de matériaux de construction, elle est le seul meuble qui montre les altérations subies au cours du temps. Sa présentation au public comme "témoin archéologique", sans intervention de restauration autre qu'une stabilisation de la surface, a donc nécessité un dispositif spécifique d'exposition. Sortie de son contexte, la table de cuisine permet de donner plus de cohérence aux espaces restaurés de la demeure.

La présentation actuelle évoque une habitation familiale au confort moderne. La restitution des décors permet de percevoir la hiérarchisation des espaces entre les pièces de réception et les zones de service. Le raffinement des meubles du boudoir (fig. 10), de la chambre et de la salle à manger des enfants contraste avec le mobilier métallique de la cuisine, conçu pour être facile à entretenir et fonctionnel. Le mobilier participe également à la mise en volume de l'espace pour garder à l'esprit l'usage des pièces adapté aux besoins des occupants. De nombreux meubles comportent des dispositifs d'articulation astucieux avec charnières et glissières pour se plier aux demandes de leurs utilisateurs. 


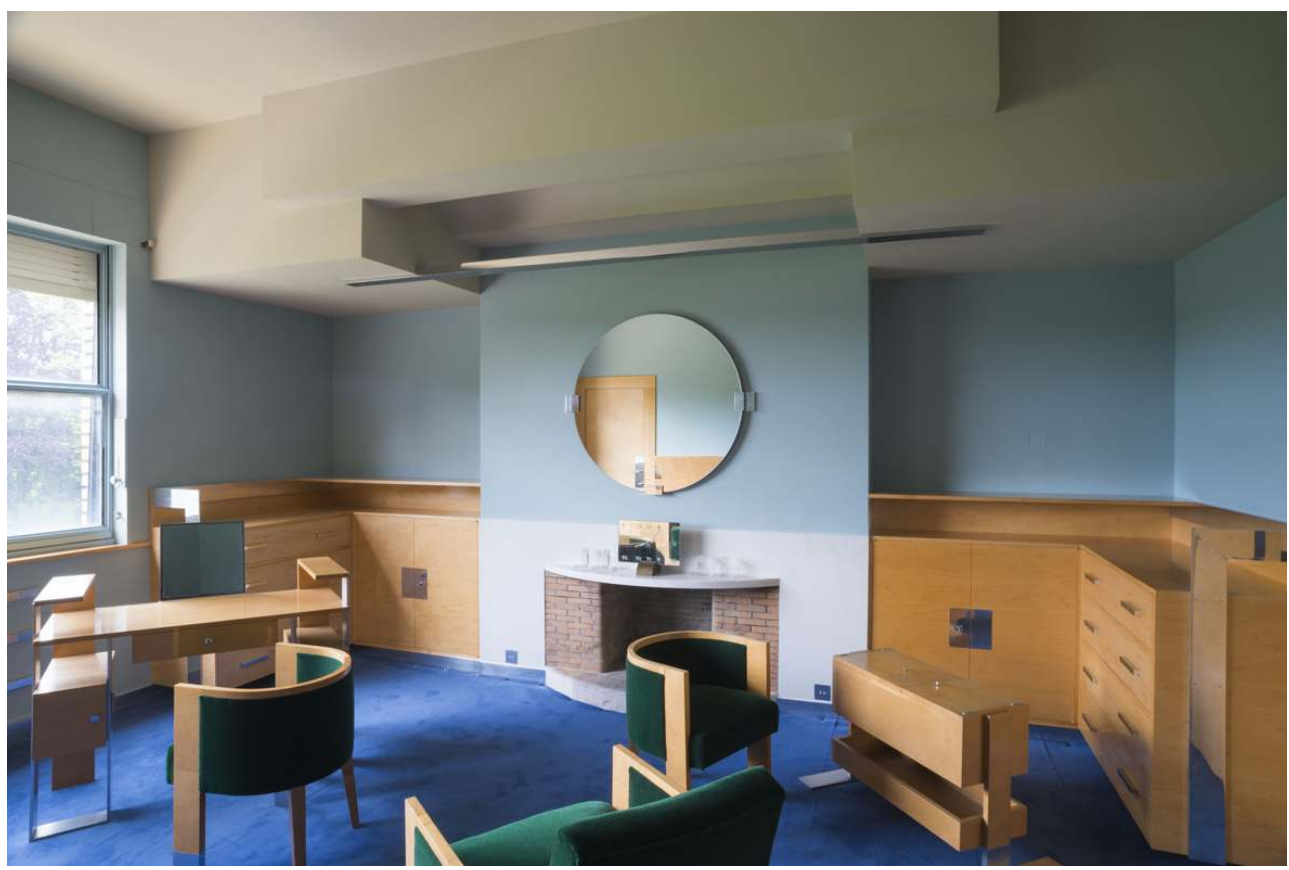

Villa Cavrois, le boudoir remeublé

Phot. Ballot, Jean-Christophe/Centre des monuments nationaux. (C) Jean-Christophe Ballot, 2015.

Les intérieurs offrent une grande harmonie, si bien qu'il est devenu parfois difficile de distinguer les éléments originaux des éléments restitués à l'identique. L'exemple le plus significatif est celui des appliques du vestibule. Trois d'entre elles sont les œuvres originales de Le Chevallier et Koechlin, la quatrième, qui manquait, a été réalisée sur le modèle des appliques d'origine. L'illusion d'ensemble est parfaite et s'accorde au plus près avec l'état historique de 1932. Une application numérique sur tablette tactile permet aux visiteurs de visualiser en réalité augmentée certaines pièces meublées telles qu'elles apparaissent sur les photographies anciennes et d'indiquer le mobilier d'origine parmi les décors reconstitués ${ }^{31}$. Ce dispositif est complété par la diffusion d'un film dans le garage qui explique la démarche de restauration. L'ancienne chambre des garçons, au premier étage, a été conservée dans son état d'abandon comme pièce " témoin ».

31 La restauration des collections s'est donc faite en adéquation avec le travail de restitution des aménagements intérieurs, notamment pour le garnissage des sièges. Afin de parvenir à un état de présentation cohérent, le velours vert choisi pour la garniture des fauteuils du boudoir d'origine est identique au textile qui recouvre la banquette restituée. Dans la chambre parentale, le satin blanc des fauteuils d'origine s'accorde avec le satin beige du lit restitué et les doubles rideaux. En l'absence de vestiges, la réfection textile s'est appuyée sur les descriptions anciennes. L'étude des matières et des couleurs utilisées dans les années 1930 ont fourni l'occasion de faire des essais comparatifs en situation à partir d'échantillons pour se rapprocher des tissus d'origine. En revanche, aucune intervention de restauration n'a été effectuée sur les surfaces en bois des meubles dont l'état de conservation s'est révélé satisfaisant. 


\section{Nouvelles perspectives}

Grâce à la vente de la collection Utterberg le 16 décembre 2015 à New York ${ }^{32}$, douze nouvelles pièces de mobilier ont rejoint les collections de la villa Cavrois. Formant deux ensembles distincts qui appartiennent au mobilier du hall et du bureau du maître de maison, ces acquisitions complètent l'ameublement de ces deux pièces jusqu'à présent vide de tout mobilier. Le premier ensemble se compose de deux tables carrées en noyer à double plateau et huit fauteuils à piétement de forme cylindrique qui affichent leur simplicité dans le traitement des volumes. Conçu pour le hall de la villa, ce mobilier répond aux exigences de confort d'une pièce destinée à la réception. Le deuxième ensemble comprend deux chaises réalisées pour le bureau de Paul Cavrois. Les formes géométriques du piétement de section carrée renvoient à une logique constructive propre à Mallet-Stevens.

$\mathrm{Au}$ même moment, l'acquisition d'un bureau avec ses deux chaises (fig. 11) en chêne cérusé se concrétise auprès d'une galerie parisienne. Réalisé pour une des deux salles d'étude, située au deuxième étage de la villa, le bureau composé d'un plateau en « $\mathrm{V}$ » et de tiroirs intégrés s'adapte aux besoins des enfants Cavrois pour lesquels il a été conçu. À l'origine, ces deux pièces, aménagées pour les filles, d'un côté, et les garçons, de l'autre étaient identiques, seules les couleurs des murs changeaient. La présentation de ces meubles nouvellement acquis permet d'intégrer une pièce supplémentaire au parcours de visite et constitue une avancée significative vers la réaffectation de l'ensemble mobilier dispersé de la villa.

Figure 11

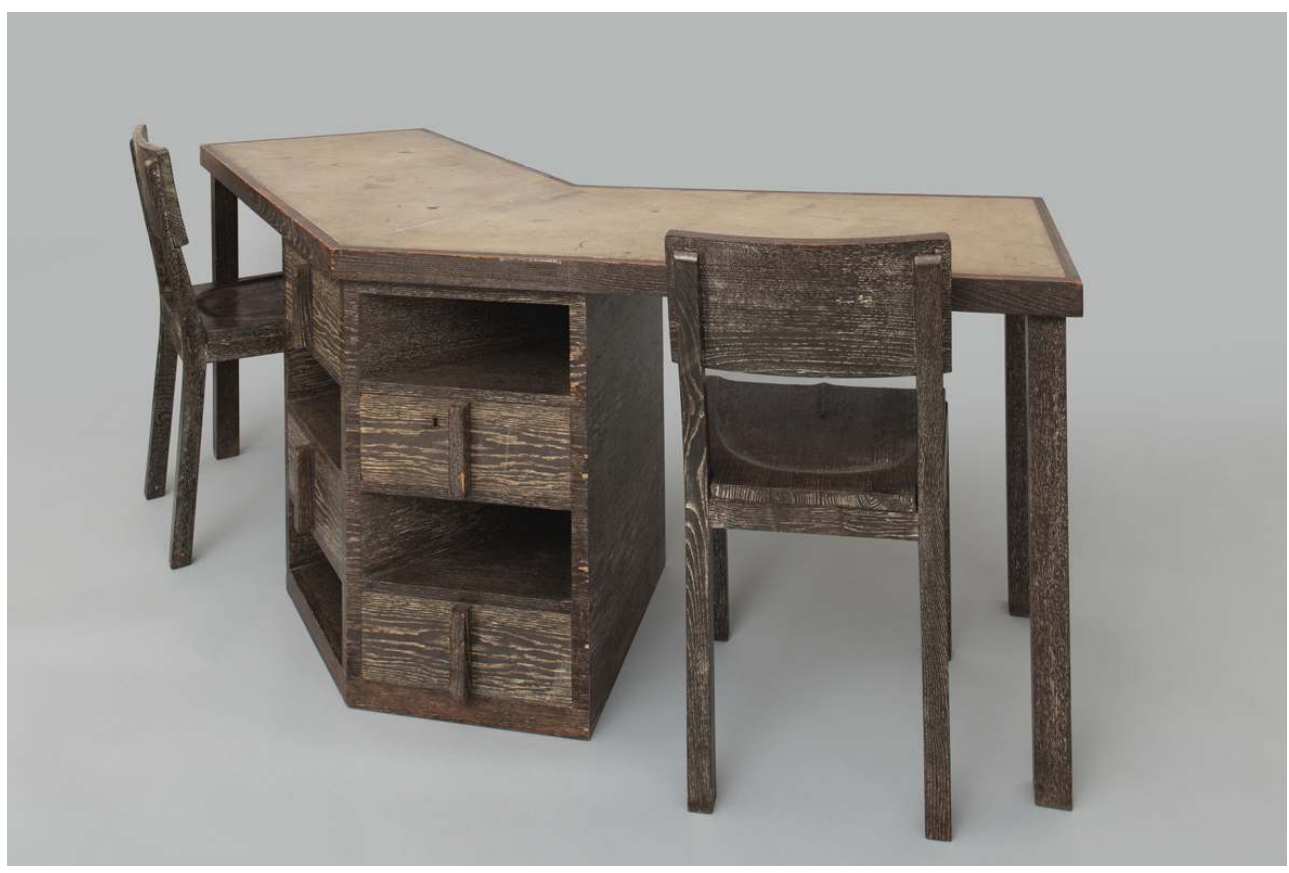

Villa Cavrois, le bureau et ses deux chaises de la salle d'étude.

Phot. Paillé, Jean-Luc/Centre des monuments nationaux. (c) Jean-Luc Paillé, 2015.

Avec cette nouvelle dynamique et le succès rencontré auprès du public dès son ouverture ${ }^{33}$, l'avenir de la villa Cavrois se présente sous des hospices heureux. L'enrichissement des 
collections se poursuit pour compléter l'ameublement des pièces et rendre davantage perceptible l'atmosphère familiale de la demeure. En parallèle, la réflexion sur la protection au titre des monuments historiques des collections est engagée avec la DRAC Nord-Pas-de-Calais-Picardie.

\section{Épilogue}

Sauvée d'une destruction programmée, la villa Cavrois offre aujourd'hui à nouveau l'image d'une demeure emblématique des années 1930 aux intérieurs équipés des dernières technologies de l'époque et aux décors soignés. Les ensembles mobiliers, nouvellement reconstitués, illustrent la relation déterminante entre intérieur et extérieur selon laquelle chaque détail répond à une logique d'harmonie et d'usage. L'habitat nouveau conçu pour la villa repose sur la conception d'un mobilier adapté à un mode de vie, à la perception des espaces, au choix et à la mise en œuvre des matériaux qui affichent une parfaite continuité avec l'architecture. Il montre aussi combien la perfection naît de la somme des détails en contribuant grandement au principe d'unité revendiqué par Mallet-Stevens où «maison, jardin, décoration et meubles sont l'œuvre d'une même personne ${ }^{34} »$.

\section{NOTES}

1. - MALLET-STEVENS, Robert. Une Demeure 1934. Boulogne: Architecture d'aujourd'hui, 1934. Réédition, Paris : Jean-Michel Place, 2000.

2. - Voir le site : www.villa-cavrois.fr [consulté le 18/05/2016].

3. - JOLY, Pierre. "La villa C à Croix ». Dans PINCHON, Jean-François (dir.). Rob Mallet-Stevens : architecture, mobilier, décoration. Paris: Action artistique/Philippe Sers, 1986, p. 48. Pierre Joly compare la villa à " un château dont la singularité tenait en un seul mot : il était moderne ».

4. - KLEIN, Richard. Robert Mallet-Stevens, la villa Cavrois. Paris : Picard, 2005, p. 113-114.

5. - Voir dans la base Mérimée : notice IA59001908.

6. - Chiffres clés : Superficie totale de la villa : $2800 \mathrm{~m}^{2}$; Superficie du parc actuel : $17600 \mathrm{~m}^{2}$; Budget global de restauration : $23 \mathrm{M} €$; Budget clos et couvert et les terrasses : 7,7 M€; Budget parc : 1,7 M€ ; Budget intérieurs : $14 \mathrm{M€}$.

7. - GOUTAL, Michel. Villa Cavrois : étude préalable à la restauration du gros-œuvre et au rétablissement $d u$ clos et du couvert, rapport de synthèse, 2002, Agence Goutal ; GOUTAL, Michel. Villa Cavrois : étude préalable à la restauration du parc, 2009, Agence Goutal.

8. - GRAFTEAUX, Benoît, KLEIN, Richard, PIJAUDIER, Joëlle. Un centre européen d'art et d'architecture, 1989, DRAC Nord-Pas-de-Calais ; MARTINEAU, Richard. Projet de réutilisation de la villa Cavrois, novembre 2004, DRAC Nord-Pas-de-Calais.

9. - Mallet-Stevens avait demandé la destruction de ses archives à sa mort.

10. - MALLET-STEVENS, Robert. Une Demeure 1934, op. cit.

11. - PINCHON, Jean-François (dir.). Rob Mallet-Stevens..., op. cit.

12. - KLEIN, Richard. Robert Mallet-Stevens, la villa Cavrois, op. cit. 
13. - CINQUALBRE, Olivier. Robert Mallet-Stevens, l'œuvre complète. Paris : éditions du Centre Pompidou, 2005.

14. - Parmi les nombreuses vues des intérieurs de la villa, les plus importantes sont celles réalisées en 1931 et 1932 par Albin Salaün.

15. - Relevés du 5 octobre 1948 et du 26 janvier 1949 (IFA 153/53/3 Cité de l'architecture et du patrimoine, Centre d'archives de l'Institut français d'architecture, Paris, fonds Pierre Barbe).

16. - Catalogue de vente, Arts décoratifs du $\mathrm{XX}^{e}$ siècle, comprenant Mobilier de Robert Mallet-Stevens provenant de la Villa C. à Croix. Sotheby's, Monaco, 5 avril 1987.

17. - « L'actualité. L'esprit Mallet-Stevens ». Gazette de l'hôtel Drouot, $1^{\mathrm{er}}$ avril 2005.

18. - Le galeriste allemand avait acheté ces ensembles en 1991 auprès de deux marchands parisiens associés, Christian Boutonnet et Rafaël Ortiz de la galerie l'Arc en Seine.

19. - Catalogues de vente, Arts décoratifs du xxe siècle. Paris, Camard, 17 juin 2003 et 9 décembre 2003.

20. - Les prix records ne permettent à cette époque aucune des préemptions envisagées pour les collections publiques.

21. - La mission de remeublement est alors confiée à Marie-Laure Jousset entre 2010 et 2013, qui est chargée du repérage des meubles et d'une réflexion sur la fabrication de reproduction de mobilier auprès de créateurs contemporains.

22. - Lots $41,42,43,44,45$, catalogue de vente, Les collections du château de Gourdon. Paris, Christie's, 29-31 mars 2011; Lots 46, 129, 328 pour la vente des 29-30 juin 2011.

23. - PORTET, Élisabeth, ROY, Clotilde. «Acquisitions de biens culturels par le Centre des monuments nationaux en 2011 - Villa Cavrois, Croix ». Monumental, 2012, Semestriel 2, p. 125.

24. - Voir le site: http://www.culturecommunication.gouv.fr/Disciplines-et-secteurs/ Monuments-historiques/Centenaire-de-la-loi-de-1913/Publications-et-bibliographies [consulté le 18/05/2016].

25. - ARCHIERI, Jean-François (dir.). Jacques Le Chevallier (1896-1987), la lumière moderne. Montreuil : Gourcuff-Gradenigo, 2007, p. 101.

26. - Lots 65, 66, catalogue de vente, Arts décoratifs du $x x^{e}$ siècle et design contemporain. Paris, Sotheby's, 6 juin 2012.

27. - Lot 63, catalogue de vente, Collection Alain Braunstein. Paris, Christie's, 23 mai 2013, p. 39.

28. - Lots 79, 80, catalogue de vente, Design moderne et contemporain. Paris, Lafon, 15 juin 2009, p. 53.

29. - GUILLAUME, Valérie. "Un certain état d'esprit moderne. Étude d'un siège en tube métallique ». Dans CINQUALBRE, O. Robert Mallet-Stevens, l'œuvre complète, op. cit., p. 62.

30. - PARSY, Paul-Hervé, PORTET, Élisabeth. «Le projet de matériauthèque ». Monumental, 2014, Semestriel 2, p. 17.

31. - Voir le site: http://www.culturecommunication.gouv.fr/Politiques-ministerielles/ Recherche-Enseignement-superieur-Technologie/Innovation-numerique/Services-numeriquesculturels-innovants/Villa-Cavrois-numerique et le site : http:// www.culturecommunication.gouv.fr/var/culture/storage/pub/

du_releve_a_la_3d_un_siecle_de_representation_du_patrimoine/index.htm [consultés le 18/05/2016].

32. - Lots 19, 20, 30, catalogue de vente, Important design. Sotheby's, New York, 16 décembre 2015.

33. - En février 2016, soit huit mois après son ouverture au public, la villa Cavrois a franchi le cap des 100000 visiteurs.

34. - MALLET-STEVENS, Robert. "La maison de M.C. à Croix (Roubaix)». Dans L'Architecture d'aujourd'hui, no 8 , novembre 1932, p. 3. 


\section{RÉSUMÉS}

La villa Cavrois, réalisée par Robert Mallet-Stevens entre 1929 et 1932 pour Paul Cavrois à Croix (Nord), est un cas exemplaire de conception homogène entre architecture, décor et mobilier. Laissée à l'abandon par son dernier propriétaire, vidée de son mobilier et vandalisée, la demeure est acquise en 2001 par l'État qui entreprend un projet global de restauration afin de " restituer » l'état historique de 1932, année de son inauguration. Différentes hypothèses de réutilisation sont envisagées, mais finalement la villa est remise en dotation au Centre des monuments nationaux en 2008 en vue de son ouverture au public. Pour retrouver l'unité des espaces, l'examen des sources documentaires et l'inventaire des collections ont permis d'identifier et de localiser le mobilier disponible afin de remeubler certaines pièces. Des ensembles mobiliers cohérents ont été acquis pour le compte de l'État à partir de 2009. L'histoire de ce projet et les choix de présentation des collections nouvellement constituées pour l'ouverture de la villa Cavrois le 13 juin 2015 sont retracées afin d'appréhender les principales étapes nécessaires à leur réalisation.

The Villa Cavrois, conceived and built at Croix (Nord) by Robert Mallet-Stevens between 1929 and 1932 for the industrialist Paul Cavrois is an exceptional example of homogeneous design between architecture, decoration and furnishings. Abandoned by its last owner, emptied of its furniture and vandalized, the mansion was finally purchased by the State in 2001. A global project was undertaken in order to restore the villa to its historical condition, set at 1932, the year it was opened. Various projects for future uses of the building were discussed but in 2008, the Villa was finally entrusted to the Centre des Monuments Nationaux in view to opening the villa to the public. To recover the unity of the villa's spatial organisation, documentary sources and an inventory of collections enabled the identification and location of surviving pieces of furniture that could be used to refurnish some of the rooms. Coherent ensembles of furniture have been purchased by the State since 2009. The history of this project and the choices made for the presentation of the newly established collections for the opening of the villa Cavrois (13 June 2015) are presented here to offer better understanding of the main steps necessary for their achievement.

\section{INDEX}

Keywords : Villa Cavrois, Mallet-Stevens, 1930s, architecture, Pierre Barbe, Croix (Nord), Centre des monuments nationaux, historic monuments, refurnishing, materials library, yellow brick, Château de Gourdon's collection, Braunstein collection, Martel brothers, Marcel Breuer, metal furniture, ministry of Culture and Communication

Mots-clés : Villa Cavrois, Mallet-Stevens, années 1930, architecture, Pierre Barbe, Croix, Centre des monuments nationaux, monuments historiques, remeublement, matériauthèque, brique jaune, collection du château de Gourdon, collection Braunstein, Frères Martel, Marcel Breuer, mobilier métallique, ministère de la Culture et de la Communication, Union des artistes modernes (UAM), collection Utterberg 


\section{AUTEUR}

\section{ÉLISABETH PORTET}

Chargée d'inventaire des collections, Centre des monuments nationaux, direction de la Conservation des monuments et des collections elisabeth.portet@monuments-nationaux.fr 